\title{
Calculation of Yield during Rewinding in the Process of Manufacturing Endless Yarn
}

\author{
By Hiroshi Kishida and Kazuhiro Nagao, Members, TMSJ \\ Nippon Rayon Co., Ltd, Uji Plant, Uji, Kyoto \\ Based on Journal of the Textile Machinery Society of Japan, Transactions, 18, T568-571 (1965) \\ abstract \\ This article shows how to calculate the yields of products which are classified by \\ rewinding quantity into grades $A, B$ and $C$ and how to increase the yield of grade $A$. \\ Used as the basis of calculation is the relation between (1) the statistical mean values \\ and the standard deviation of a group of inputs during winding and (2) statistical mean \\ values and standard deviations of final products. \\ It is difficult to lessen the standard deviation of the winding quantity of a group \\ of inputs or Grade $A$ in a group of outputs, but it is easy to change the mean wind- \\ ing quantity of a group of inputs. Therefore, by fixing the standard deviations we can \\ formulate the relation between the yield of each grade and the winding quantity of a \\ group of inputs and decide the most economical conditions for the rewinding process.
}

\section{Introduction}

In textile manufacturing, especially in the continuous processes of manufacturing yarn-like products, there are many cases where a semi-processed product having a specified length or weight is divided by rewinding into several products. This article gives a formula for calculating their yields. These calculations may be used even if such articles transform during the manufacturing process. For example, the calculations may be used even if roving yarn transforms into spun yarn or undrawn yarn into drawn yarn. We define the primary group of articles as "the inputs" and the secondary group as "the outputs."

The input and output groups each consist of large numbers. The distribution of each input is a simple statistical one. The outputs contain short pieces which are fractions of the weight of the inputs. In other words, the outputs contain (1) full-wound, final products (which we call here Grade $A$ ); (2) the remainder, i.e., waste remaining after a specified quantity of Grade $A$ is collected from the inputs (this we call Grade $C$ ); and (3) products which are between Grades $A$ and $C$ and which we call Grade $B$.

The distribution of Grade $A$ is a simple statistical one. This article presents a formula for calculating, during rewinding, the yields of Grades $A, B$ and $C$ from inputs having the simple statistical distribution.

As an applied example, fix the standard deviations of inputs and Grade $A$ and the mean value of Grade $A$. Then the yields of Grades $A, B$ and $C$ are calculable against the mean value of inputs.

\section{Assumptions and Nomenclatures}

The following assumptions are made as a preliminary to calculation :

(a) That the distributions of the wound quantities of the inputs and of Grade $A$ are normal.

(b) That, during the manufacturing process, there is nothing to cut, except yarn for sampling for grading $A$.

(c) That the regions further from the mean value by $\pm 3 \sigma$ are negligible.

(d) That the wound quantities of inputs and Grade $A$ are independent functions of each other.

The following nomenclatures are used:

$Q_{A U}, Q_{A L}:$ Upper and lower limits of Grade $A$., the final designation.

$Q_{c}$ : Upper limit of waste, Grade $C$.

$W, \bar{W}:$ quantity and mean value of the inputs.

$x, \bar{x}$ : quantity and mean value of Grade $A$.

$X, \bar{X}:$ quantity and mean value of the remainder of the inputs after $(N-1)$ of Grade $A$ is collected from one input.

$N$ : mean number of Grade $A$ collected from one input.

$\sigma_{W}:$ standard deviation of the inputs.

$\sigma x$ : standard deviation of Grade $A$. 
$\sigma x:$ standard deviation of $X$.

$F(X)$ : density function of $X$.

$f(x)$ : density function of $x$.

$E(A), E(B), E(C)$ : expected statistical values of Grades $A, B$ and $C$ collected from the remainder $X$.

$E[A], E[B] \cdots$ expected statistical values of yields of Grades $A$ and $B$.

$E[C]$...expected statistical value of the yield of Grade $C$, waste.

\section{Calculating Grade A}

The mean value $\bar{X}$, the standard deviation $\sigma x$, the density function $F(X)$ of the remain $X$ and the density function $f(x)$ are shown below:

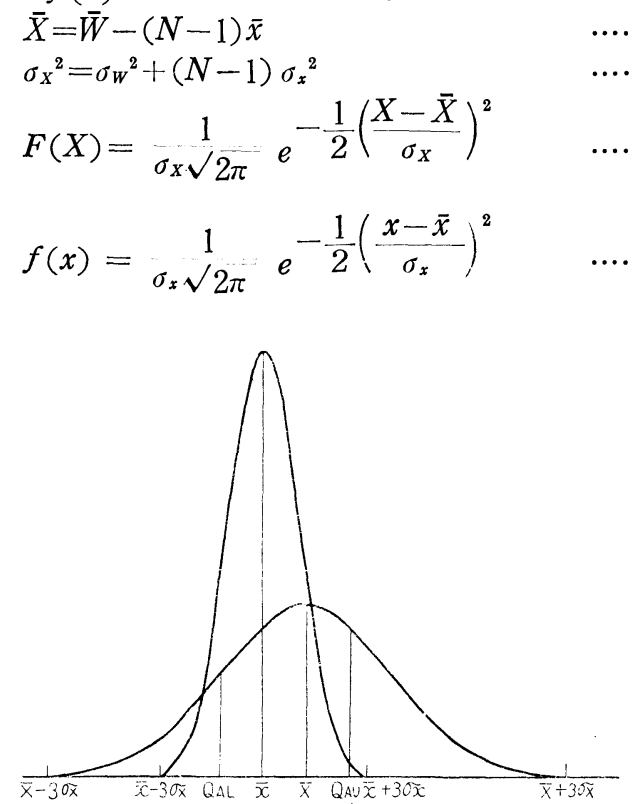

Fig. 1 Distribution of $f(x), F(X)$

Fig. 1 shows the distributions of $x$ and $X$, but the quantitative order of $\bar{X}-3 \sigma_{x}, \bar{x}-3 \delta_{x}, Q_{A L}, \bar{x}, \bar{X}$, $\bar{x}+3 \sigma_{x}$ and $\bar{X}+3 \sigma_{x}$ is changeable according to practical calculation, and then the integral limits must change accordingly. The integral limits in this article are based on the calculations described later. The distribution curves in Fig. 1 are divided into several regions :

(i) in the region $X<\bar{X}-3 \sigma_{X}, \quad F(X)=0$, i.e., $E(A)=0, E(B)=0, E(C)=0$.

(ii) in the region $\bar{X}-3 \sigma_{X} \leqq X<Q_{A L}, \quad E(A)=0$, $E(B) \neq 0, \quad E(C) \neq 0$.

(iii) in the region $Q_{A L} \leqq X \leqq \bar{X}+3 \sigma_{X}, \quad E(A) \neq 0$, $E(B) \neq 0, \quad E(C) \neq 0$. (iv) in the region $\bar{X}+3 \sigma_{X}<X, F(X)=0$, i.e., $E(A)$ $=0, E(B)=0, E(C)=0$.

The yield $E[A]$ of Grade $A$, is shown thus :

$$
E[A]=\frac{1}{\bar{W}}\{(N-1) \bar{x}+E(A)\}
$$

In the region $Q_{A L} \leqq X \leqq \bar{X}+3 \sigma_{X}$,

$$
E(A)+E(B)+E(C)=\int_{Q_{A L}}^{\bar{X}+3 \sigma_{X}} X F(X) d X
$$

and

$$
\begin{aligned}
E(B)+E(C) & =\int_{Q_{A L}}^{\bar{X}+3 \sigma_{X}} F(X) \int_{\bar{x}-3 \sigma_{x}}^{\bar{X}}(X-x) f(x) d x d X \\
& +\int_{Q_{A L}}^{\bar{X}+3 \sigma_{X}} F(X) \int_{\bar{x}-3 \sigma_{x}}^{Q_{A L}} x f(x) d x d X \\
& +\int_{Q_{A U}}^{\bar{X}+3 \sigma_{X}} F(X) \int_{Q_{A U}}^{\bar{x}+3 \sigma_{x}} x f(x) d x d X \ldots \ldots \ldots
\end{aligned}
$$

Therefore,

$$
\begin{aligned}
E(A) & =\int_{Q_{A L}}^{\bar{X}+3 \sigma_{X}} X F(X) d X \\
& -\int_{Q_{A L}}^{\bar{X}+3 \sigma_{X}} F(X) \int_{\bar{x}-3 \sigma_{x}}^{X}(X-x) f(x) d x d X \\
& -\int_{Q_{A L}}^{\bar{X}+3 \sigma_{X}} F(X) \int_{\bar{x}-3 \sigma_{x}}^{Q_{A L}} x f(x) d x d X \\
& -\int_{Q_{A U}}^{\bar{X}+3 \sigma_{X}} F(X) \int_{Q_{A U}}^{\bar{x}+3 \sigma_{x}} x f(x) d x d X
\end{aligned}
$$

Eq. (7) is $E(A)$ in Eq. (5), because $E(A)$ is absent from the other regions.

\section{Yield of Grade $C$, Waste}

Think of it as the same as the yield of Grade A and divided it into 5 regions :

(i) in the region $X<\bar{X}-3 \sigma_{X}, \quad F(X)=0$, i.e., $E(A)$ $=0, E(B)=0, E(C)=0$.

(ii) in the region $\bar{X}-3 \sigma_{X} \leqq X<\bar{x}-3 \sigma_{x}, \quad E(A)=0$, $E(B) \neq 0, E(C)=0$.

(iii) in the region $\bar{x}-3 \sigma_{x} \leqq X \leqq \bar{x}+3 \sigma_{x}+Q c, E(A) \neq$ $0, E(B) \neq 0, \quad E(C) \neq 0$.

(iv) in the region $\bar{x}+3 \sigma_{x}+Q_{c}<X \leqq \bar{X}+3 \sigma_{X}, \quad E(A)$ $\neq 0, \quad E(B) \neq 0, \quad E(C)=0$.

(v) in the region $\bar{X}+3 \sigma_{X}<X, F(X)=0$, i.e., $E(A)$ $=0, E(B)=0, E(C)=0$.

The yield of Grade $C$, then, is : 


$$
E[C]=\frac{E(C)}{\bar{W}}
$$

$E(C)$ is present only in the rigion $\bar{x}-3 \sigma_{x} \leqq X \leqq \bar{x}+3 \sigma_{x}$ $+Q_{c}$.

$E(C)$ is shown thus:

$$
E(C)=\int_{\bar{x}-3 \sigma_{x}}^{\bar{x}+3 \sigma_{x}+Q_{c}} F(X) \int_{X-Q_{c}}^{X}(X-x) f(x) d x d X \cdots(9)
$$

Eq. (9) corresponds to $E(C)$ in Eq. (8).

\section{Calculating the Yield of Grade $B$}

The yield of Grade $B$ is shown by Eq. (10).

$$
E[B]=\{1.00-(E[A]+E[C])\}
$$

\section{Development of Equations}

It is possible to calculate the yields of Grades $A$, $B$ and $C$ by using Eqs. (5), (7), (8), (9) and (10).

Here we show the methods to calculate

$$
\begin{aligned}
& \int_{X} X F(X) d X \quad \text { in Eq. (7) and } \int_{X} F(X) \\
& \int_{x}(X-x) f(x) d x d X \text { in Eqs.(7) and (9). The }
\end{aligned}
$$

3rd and 4th items of Eq. (7) are treated in the same way as the 2 nd item.

Consider the following functions :

$$
\frac{X-\bar{X}}{\sigma_{X}}=T \quad \frac{x-\bar{x}}{\sigma_{x}}=t
$$

$T(Q A L)$, if expressed as the value of $T$ in $X=Q_{A L}$, transforms itself into:

$$
\begin{aligned}
\int_{Q_{A L}}^{\bar{X}+3 \sigma_{X}} X F(X) d X & \int_{Q_{A L}}^{\bar{X}+3 \sigma_{X}} \frac{X}{\sigma_{X} \sqrt{ } 2 \pi} e^{-\frac{1}{2}\left(\frac{X-\bar{X}}{\sigma_{X}}\right)^{2}} d X \\
= & \int_{T\left(\bar{X}+3 \sigma_{X}\right)}^{T} \frac{1}{\sqrt{ } 2 \pi} e^{-\frac{1}{2} T^{2}} d T+\frac{\sigma_{X}}{\sqrt{ } 2 \pi} \\
& \int_{T(Q A L)}^{T\left(\bar{X}+3 \sigma_{X}\right)} T^{-\frac{1}{2} T^{2}} d T \\
& =\bar{X} \int_{T(Q A L)}^{T\left(\bar{X}+3 \sigma_{X}\right)} e^{1} e^{-\frac{1}{2} T^{2}} d T
\end{aligned}
$$

$$
+\frac{\sigma_{X}}{\sqrt{ } 2 \pi}\left(e^{{ }_{2}^{2} T^{2}}\right]_{T(Q A L)}^{T\left(X+3 \sigma_{X}\right)}
$$

The 1 st item of Eq. (11) is calculable from the "Table of normal distribution." As to the calculation of $\int_{X} F(X) \int_{x}(X-x) f(x) d x d X:$

$$
I=\int_{X} \frac{1}{\sigma_{X} \sqrt{2} \pi} e^{-\frac{1}{2}\left(\begin{array}{c}
X-\bar{X} \\
\sigma_{X}
\end{array}\right)^{2}}
$$

$$
\begin{aligned}
& \int_{x}^{(X-x)} \frac{1}{\sigma_{x} \sqrt{ } 2 \pi} e^{-\frac{1}{2}\left(\frac{x-\bar{x}}{\sigma_{x}}\right)^{2}} d x d X \\
= & \int_{X} \underset{\sigma_{X} \sqrt{ } 2 \pi}{X} e^{-\frac{1}{2}\left(\frac{X-\bar{X}}{\sigma_{X}}\right)^{2} \cdot}
\end{aligned}
$$

$$
\begin{aligned}
& \int_{x} \frac{1}{\sigma_{x} \sqrt{ } 2 \pi} e^{-\frac{1}{2}\left(\frac{x-\bar{x}}{\sigma_{X}}\right)^{2}} \cdot d x d X \\
& -\int_{X} \int_{\sigma_{X} \sqrt{ } 2 \pi^{2}}^{1} e^{-\frac{1}{2}\left(\frac{X-\bar{X}}{\sigma_{X}}\right)} \cdot
\end{aligned}
$$

$$
\int_{x}^{t_{\sigma_{x}+\bar{x}} \sigma_{x} \sqrt{ } 2 \pi} e^{-\frac{1}{2}\left(\begin{array}{c}
x-\bar{x} \\
\sigma_{x}
\end{array}\right)^{2}} d x d X
$$

$=\int_{T(X)} \frac{X-\bar{x}}{\sqrt{ } 2 \pi} e^{-1^{1} T^{2}} \int_{t(x)} \frac{1}{\sqrt{ } 2 \pi} e^{-\frac{1}{2} t^{2}} d t d T$

$-\int_{T(X)} \frac{1}{\sqrt{ } 2 \pi} e^{-\frac{1}{2} T^{2}} \int_{t(x)} \frac{t_{\sigma_{x}}}{\sqrt{ } 2 \pi} e^{-\frac{1}{2} t^{2}} d t d T$

$=\int_{T(X)}^{X-\bar{x}} e^{-\frac{1}{2} T^{2}} \int_{t(x)} \frac{1}{\sqrt{ } 2 \pi} e^{-\frac{1}{2} t^{2}} d t d T$

$-\int_{T(x)} \frac{1}{\sqrt{2 \pi}} e^{-\frac{1}{2} T^{2}} \cdot \frac{\sigma_{x}}{\sqrt{ } 2 \pi}\left[e^{-\frac{1}{2} t^{2}}\right]_{t(x)}$

$\doteqdot \frac{\Delta X}{\sigma_{X}} \sum \frac{1}{\sqrt{ } 2 \pi}(X-\bar{x}) e^{-\frac{1}{2} T^{2}} \int_{t(x)} \frac{1}{\sqrt{ } 2 \pi} e^{-\frac{1}{2} t^{2}} d t$

$+\frac{\Delta X}{\sigma_{X}} \sum \frac{\sigma_{x}}{2 \pi} e^{-\frac{1}{2} T^{2}}\left[e^{-\frac{1}{2} t^{2}}\right] t(x)$ 
This equation is calculable by applying the principle of divisional measurement and making the condition $\Delta X$ small enough.

\section{Examples of Calculation}

Since it is difficult to diminish the standard deviation of the inputs or of Grade $A$, as the most practical example, we must fix the winding quantities of the inputs in such a way as to get the maximum yield without changing the specified winding quantities of Grade $A$. For this purpose, we show the relation between the yields of Grades $A, B$ and $C$ and the mean quantity of inputs.

The yields of Grades $A, B$ and $C$ relative to the mean value of inputs are calculable by substituting the quantities $\bar{x}=930, \sigma_{x}=10, Q_{A U}=1000, Q_{A L}=860, Q_{C}=$ 60, $N=2, \sigma_{W}=153\left(\sigma_{x}=158\right)$. Calculate $E\lceil A]$ and $E[C]$ first, when the mean quantity of the input

$$
\bar{W}=N \bar{x}=1860(\bar{X}=930) \text {. }
$$

Then omit the 3rd and 4th items of Eq. (7). The result :

$$
\begin{aligned}
& E(A)=\int_{860}^{1414} F(X) d X \\
& -\int_{860}^{1414} F(X) \quad \int_{900}^{X}(X-x) f(x) d x d X \\
& =\int_{T(860)}^{T(1414)} F(T) d T-\frac{\Delta X}{\sigma_{X}} \\
& \sum_{860 \leqq X \leqq 1414} \frac{1}{\sqrt{2 \pi}}(X-\bar{x}) e^{-{ }_{2}^{1} T^{2}} \\
& \int_{t(900)}^{t(X)} \frac{1}{\sqrt{ } 2 \pi} e^{-\frac{1}{2} t^{2}} d t-\frac{\Delta X}{\sigma_{X}} \\
& \sum_{860 \leqq X \leqq 1414} 2 \pi e^{\sigma_{x}} e^{-\frac{1}{2} T^{2}} \\
& {\left[e^{-\frac{1}{2} t^{2}}\right]_{t(900)}^{t(X)}}
\end{aligned}
$$

where $t(900)=(900-930) / 10$.

Divide, region $860 \leqq X \leqq 1414$ into 554 sections by $\Delta X=1$, then calculate $E(A)$ and insert the result into Eq. (5).

Similary, $E[C]$, in the light of Eq. (8), is :

$$
\begin{aligned}
& E(C)=\int_{900}^{1020} F(X) \int_{X-60}^{X}(X-x) f(x) d x d X \\
& ={ }_{\sigma_{X}}^{\Delta X} \sum_{900 \leqq X \leqq 1020} \frac{1}{\sqrt{ } 2 \pi}(X-\bar{x}) e^{-{ }_{2}^{1} T^{2}} \\
& \int_{t(X-60)}^{t(X)} \frac{1}{\sqrt{ } 2 \pi} e^{-\frac{1}{2} t^{2}} d t
\end{aligned}
$$

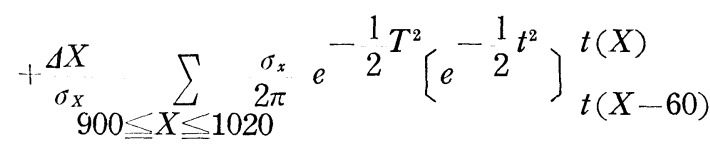

By the same method, rigion $900 \leqq X \leqq 1020$ is divided into 120 sections by $\Delta X=1$, then $E[C]$ is calculated by inserting this $E(C)$ into Eq. (8).

$E[A], E[B]$ and $E[C]$ are similarly calculated

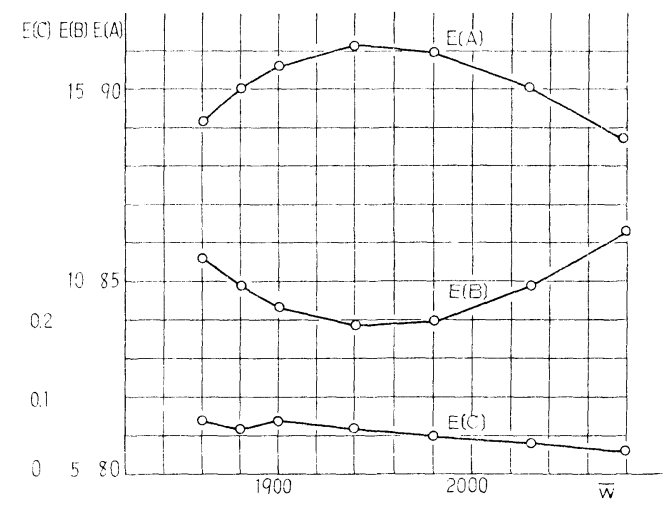

Fig. 2 The yield of each clasified grade against the winding quantity of the input

against $\bar{W}=1880,1900,1940,1980,2030$ and 2080 . Fig. 2 shows the relation between the yields and the winding mean value of the input.

From this figure we find that the yield of Grade $C$ is negligible beside any quantity of $\bar{W}$; and that the maximum yield of Grade $A$ is obtainable if $\bar{W}$ is about 1940.

\section{Conclusions}

No general conculusion is deducible because this practical calculation differs in each case. However, we can calculate the expected statistical value of the yields by putting the mean quantities and the standard deviations of the input and Grade $A$. For example, we have shown that it is possible to increase the yield, without extra trouble, by choosing the mean quantity of the input, even where it is not possible to diminish the standard deviation of winding quantity 